\title{
Electromagnetic Scattering of Finite and Infinite 3D Lattices in Polarizable Backgrounds
}

\author{
Benjamin Gallinet and Olivier J. F. Martin \\ Nanophotonics and Metrology Laboratory, Swiss Federal Institute of Technology Lausanne (EPFL), \\ 1015 Lausanne, Switzerland
}

\begin{abstract}
A novel method is elaborated for the electromagnetic scattering from periodical arrays of scatterers embedded in a polarizable background. A dyadic periodic Green's function is introduced to calculate the scattered electric field in a lattice of dielectric or metallic objects. The method exhibits strong advantages: discretization and computation of the field are restricted to the volume of the scatterers in the unit cell, open and periodic boundary conditions for the electric field are included in the Green's tensor, and finally both near and far-fields physics are directly revealed, without any additional computational effort. Promising applications include the design of periodic structures such as frequency-selective surfaces, photonic crystals and metamaterials.
\end{abstract}

Keywords: Electromagnetic Scattering, Dyadic Green Function, Periodic Structures

PACS: $42.70 . \mathrm{Qs}, 78.67 . \mathrm{Bf}, 02.70 . \mathrm{Pt}$

Recent developments in the fabrication of periodic structures with tailored optical periodic structures call for specific modeling tools. Most real structures are threedimensional but still very few techniques are able to simulate them without considerable computational efforts $[1,2]$. There is therefore a need to seek for a general method supporting full 3D vectorial calculations, enabling the investigation of both near and far-fields physics. Our approach is based on the volume integral equation for scattering in free space and at surfaces [3].

We first develop the Bloch modes calculations in lattices using the Green's tensor formalism. The method exhibits strong advantages, especially about the restriction of the discretization and the minimal computational efforts required to reveal both near and far-field physics. Then two examples of calculations are reported to illustrate some physical effects that can be simulated: grating effects, propagation in multi-dimensional finite and infinite size lattices. Promising applications include the design of periodic structures such as frequency-selective surfaces, photonic crystals, and metamaterials.

\section{DYADIC GREEN'S FUNCTION FOR PERIODIC STRUCTURES}

The scalar Green's function has been previously reported for energy bands calculations using Schrödinger's equation in a periodic potential [4]. Our objective is to deduct its dyadic form in electromagnetism for a lattice of point sources. Consider a scattering system described by a dielectric function $\varepsilon(\mathbf{r})$ embedded in an infinite homogeneous background medium $\varepsilon_{B}$. Nonmagnetic materials and harmonic fields with an $\exp (-i \omega t)$ dependance are assumed. When this system is illuminated by an incident field $\mathbf{E}^{0}(\mathbf{r})$ propagating in the background medium, the total electric field (incident plus scattered field) is a solution of the vectorial wave equation:

$$
\nabla \times \nabla \times \mathbf{E}(\mathbf{r})-k_{0}^{2} \varepsilon(\mathbf{r}) \mathbf{E}(\mathbf{r})=0,
$$

where $k_{0}^{2}=\omega^{2} / c^{2}$ is the vacuum wave number. The permittivity $\varepsilon(\mathbf{r})$ is assumed to have a spatial periodicity: $\varepsilon(\mathbf{r}+\mathbf{l})=\varepsilon(\mathbf{r})$, where $\mathbf{I}=n \mathbf{a}_{1}+m \mathbf{a}_{2}+p \mathbf{a}_{3}(m, n, p$ integers) is a translation vector of the lattice and $\mathbf{a}_{1}, \mathbf{a}_{2}, \mathbf{a}_{3}$ are the translation primitive vectors. The volume of the unit cell is called $\Omega$. If the dielectric landscape is anisotropic, one can simply replace $\varepsilon(\mathbf{r}) \mathbf{E}(\mathbf{r})$ by the contraction $\underline{\underline{\varepsilon}}(\mathbf{r})$. $\mathbf{E}(\mathbf{r})$ in the following. The solutions of Eq. (1) are Bloch modes $\mathbf{E}_{\mathbf{k}}(\mathbf{r})$ satisfying the following translational symmetry property

$$
\mathbf{E}_{\mathbf{k}}(\mathbf{r}+\mathbf{l})=e^{i \mathbf{k} \cdot \mathbf{l}} \mathbf{E}_{\mathbf{k}}(\mathbf{r}),
$$

where $\mathbf{k}$ is the Bloch vector characterizing the modes symmetry and lying in the first Brillouin Zone (1BZ). The dielectric contrast is given by $\Delta \varepsilon(\mathbf{r})=\varepsilon(\mathbf{r})-\varepsilon_{B}$ and carries the same periodicity as $\varepsilon(\mathbf{r})$. We can now rewrite Eq. (1) as an inhomogeneous equation:

$$
\nabla \times \nabla \times \mathbf{E}(\mathbf{r})-k_{B}^{2} \mathbf{E}(\mathbf{r})=k_{0}^{2} \Delta \varepsilon(\mathbf{r}) \mathbf{E}(\mathbf{r}),
$$

where $k_{B}^{2}=k_{0}^{2} \varepsilon_{B}$. The incident field $\mathbf{E}^{0}(\mathbf{r})$ is a solution of the corresponding homogeneous equation:

$$
\nabla \times \nabla \times \mathbf{E}^{0}(\mathbf{r})-k_{B}^{2} \mathbf{E}^{0}(\mathbf{r})=0 .
$$

To compute $\mathbf{E}_{\mathbf{k}}(\mathbf{r})$, let us introduce the dyadic Green's function $\underline{G}_{\mathbf{k}}\left(\mathbf{r}, \mathbf{r}^{\prime}\right)$ as a solution of the vector wave equation [Eq. (1)] with a point source term for any $\mathbf{r}, \mathbf{r}^{\prime}$ in the 
unit cell $\Omega$ :

$$
\nabla \times \nabla \times \underline{\underline{G}}_{\mathbf{k}}\left(\mathbf{r}, \mathbf{r}^{\prime}\right)-k_{B}^{2} \underline{\underline{G}}_{\mathbf{k}}\left(\mathbf{r}, \mathbf{r}^{\prime}\right)=\underline{\underline{\mathbf{1}}} \delta\left(\mathbf{r}-\mathbf{r}^{\prime}\right),
$$

and also satisfying:

$$
\begin{aligned}
\underline{\underline{G}}_{\mathbf{k}}\left(\mathbf{r}, \mathbf{r}^{\prime}\right) & =\underline{\underline{G}}_{\mathbf{k}}^{*}\left(\mathbf{r}^{\prime}, \mathbf{r}\right), \\
\underline{\underline{G}}_{\mathbf{k}}\left(\mathbf{r}+\mathbf{l}, \mathbf{r}^{\prime}\right) & =\underline{\underline{1}} e^{i \mathbf{k} \cdot \mathbf{l}} \cdot \underline{\underline{G}}_{\mathbf{k}}\left(\mathbf{r}, \mathbf{r}^{\prime}\right),
\end{aligned}
$$

where $\mathbf{k}$ is in the $1 \mathrm{BZ}$ and $\underline{\underline{1}}$ is the $3 \times 3$ identity matrix. $\underline{\underline{G}} \mathbf{k}\left(\mathbf{r}, \mathbf{r}^{\prime}\right)$ has transformation properties of a dyadic [5] and is given by:

$$
\underline{\underline{G}} \mathbf{k}\left(\mathbf{r}, \mathbf{r}^{\prime}\right)=\sum_{\mathrm{I}} \underline{\underline{G}}_{0}\left(\mathbf{r}-\mathbf{l}, \mathbf{r}^{\prime}\right) e^{i \mathbf{k} \cdot \mathbf{l}}
$$

where $\underline{\underline{G}}_{0}\left(\mathbf{r}, \mathbf{r}^{\prime}\right)$ is a solution of Eq. (5) in the unit cell [3]:

$$
\underline{\underline{G}} 0\left(\mathbf{r}, \mathbf{r}^{\prime}\right)=\left(\underline{\underline{1}}+\frac{\nabla \nabla}{k_{B}^{2}}\right) \frac{e^{i k_{B}\left|\mathbf{r}-\mathbf{r}^{\prime}\right|}}{4 \pi\left|\mathbf{r}-\mathbf{r}^{\prime}\right|} .
$$

Equation (8) is numerically evaluated by truncating the infinite sum to a finite number of point sources. Its convergence can be accelerated by different techniques (such as Ewald transformations), which have been a subject of extensive research over the past years $[6,7,8]$. The spectral representation of $\underline{G}_{\mathbf{k}}\left(\mathbf{r}, \mathbf{r}^{\prime}\right)$ can be obtained by expanding its lattice-periodic part over the translation vectors $\mathbf{G}$ of the reciprocal lattice:

$\underline{\underline{G}}_{\mathbf{k}}\left(\mathbf{r}, \mathbf{r}^{\prime}\right)=\sum_{\mathbf{G}} \frac{1}{k_{B}^{2}|\Omega|} \frac{1 k_{B}^{2}-(\mathbf{k}+\mathbf{G})(\mathbf{k}+\mathbf{G})}{|\mathbf{k}+\mathbf{G}|^{2}-k_{B}^{2}} e^{i(\mathbf{k}+\mathbf{G}) \cdot\left(\mathbf{r}-\mathbf{r}^{\prime}\right)}$

where $|\Omega|$ is the unit cell volume. Here $\mathbf{G}=n \mathbf{b}_{1}+m \mathbf{b}_{2}+$ $p \mathbf{b}_{3}$ ( $m, n, p$ integers) where $\mathbf{b}_{1}, \mathbf{b}_{2}, \mathbf{b}_{3}$ are the primitive lattice vectors satisfying by definition $\mathbf{a}_{i} \cdot \mathbf{b}_{j}=\delta_{i j}, \forall i, j=$ $1,2,3$.

The Bloch function $\mathbf{E}_{\mathbf{k}}(\mathbf{r})$ solution of Eq. (3) can then be written as:

$$
\mathbf{E}_{\mathbf{k}}(\mathbf{r})=\mathbf{E}_{\mathbf{k}}^{0}(\mathbf{r})+\int_{\Omega} d \mathbf{r}^{\prime} \underline{\underline{G}} \mathbf{k}\left(\mathbf{r}, \mathbf{r}^{\prime}\right) \cdot k_{0}^{2} \Delta \varepsilon\left(\mathbf{r}^{\prime}\right) \mathbf{E}_{\mathbf{k}}\left(\mathbf{r}^{\prime}\right)
$$

where $\mathbf{E}_{\mathbf{k}}^{0}(\mathbf{r})$ is the corresponding Bloch component of the incident field $\mathbf{E}^{0}(\mathbf{r})$. To find the field at any position $\mathbf{r}$, an integration is performed on the volume of a single scatterer of dielectric contrast $\Delta \varepsilon(\mathbf{r})$ in the unit cell $\Omega$. Since $\underline{G}_{\mathbf{k}}\left(\mathbf{r}, \mathbf{r}^{\prime}\right)$ is diverging for $\mathbf{r}=\mathbf{r}^{\prime}$, the principal value of the integral in Eq. (11) is taken, and the singularity of $\underline{\underline{G}}_{\mathbf{k}}\left(\mathbf{r}, \mathbf{r}^{\prime}\right)$ treated separately:

$$
\begin{aligned}
\mathbf{E}_{\mathbf{k}}(\mathbf{r}) & =\lim _{\delta V \rightarrow 0} \int_{\Omega-\delta V} d \mathbf{r}^{\prime} \underline{\underline{G}} \mathbf{k}\left(\mathbf{r}, \mathbf{r}^{\prime}\right) \cdot k_{0}^{2} \Delta \varepsilon\left(\mathbf{r}^{\prime}\right) \mathbf{E}_{\mathbf{k}}\left(\mathbf{r}^{\prime}\right) \\
& +\mathbf{E}_{\mathbf{k}}^{0}(\mathbf{r})-\underline{\underline{L}} \cdot \frac{\Delta \varepsilon(\mathbf{r})}{\varepsilon_{B}} \mathbf{E}_{\mathbf{k}}(\mathbf{r})
\end{aligned}
$$

where the infinitesimal volume $\delta V$ centered at point $\mathbf{r}$ is used to exclude the singularity. The source dyadic $\underline{\underline{L}}$ depends on the shape of $\delta V$ and is equal to $\frac{1}{3} \underline{\underline{1}}$ for a spherical exclusion volume [9]. To solve Eq. (12) numerically, a grid with $N$ meshes over the scatterer in the primitive cell is defined, each mesh centered at position $\mathbf{r}_{i}$ and having a volume $V_{i}$. Introducing the discretized field $\mathbf{E}_{\mathbf{k}, i}=\mathbf{E}_{\mathbf{k}}\left(\mathbf{r}_{i}\right)$, dielectric contrast $\Delta \varepsilon_{i}=\Delta \varepsilon\left(\mathbf{r}_{i}\right)$, and Green's tensor $\underline{\underline{G}}_{\mathbf{k}, i, j}=\underline{\underline{G}} \mathbf{k}\left(\mathbf{r}_{i}, \mathbf{r}_{j}^{\prime}\right)$, Eq. (11) becomes a system of linear equations:

$$
\begin{aligned}
\mathbf{E}_{\mathbf{k}, i} & =\mathbf{E}_{\mathbf{k}, i}^{0}+\sum_{j=1, j \neq i}^{N} \underline{G}_{\mathbf{k}, i, j} \cdot k_{0}^{2} \Delta \varepsilon_{j} V_{j} \mathbf{E}_{\mathbf{k}, j} \\
& +\underline{\underline{M}}_{i} \cdot k_{0}^{2} \Delta \varepsilon_{i} \mathbf{E}_{\mathbf{k}, i}-\underline{\underline{L}} \cdot \frac{\Delta \varepsilon_{i}}{\varepsilon_{B}} \mathbf{E}_{\mathbf{k}, i}
\end{aligned}
$$

with

$$
\underline{\underline{M}}_{i}=\lim _{\delta V \rightarrow 0} \int_{\Omega-\delta V} d \mathbf{r}^{\prime} \underline{\underline{G}}_{0}\left(\mathbf{r}_{i}, \mathbf{r}^{\prime}\right) .
$$

The value of the self term $\underline{\underline{M}}_{i}$ is obtained by numerical quadrature on mesh $i$ [3]. Remark that the discretization of the periodic system is performed on the scatterer in the primitive cell only. The field inside the scatterer is implicitly given by Eq. (11) and must therefore be computed by solving the system of linear equations of (13), e.g. with the conjugate gradients method [10]. Open and periodic boundary conditions for the electric field are included in the Green's tensor and do not need to be explicitly treated in the computation. The field outside the scatterer is explicit and is immediately found by a matrix multiplication. The only computational effort is then in the evaluation of the lattice Green's tensor.

\section{NUMERICAL SIMULATIONS}

We first investigate a one-dimensional array of dielectric cubes in air [Fig. 1(a)]. The incoming field $\mathbf{E}^{0}(\mathbf{r})$ is a plane wave exciting only one Bloch mode of the lattice. In Fig. 1(b), the preferred directions for the normalized total field intensity $|\mathbf{E}(\mathbf{r})|^{2} /\left|\mathbf{E}^{0}(\mathbf{r})\right|^{2}$ reveal different types of scattered waves: reflected, transmitted $(m=0)$ and different orders of diffraction $m$. The nanoscale field mapping is also easily obtained without running additional calculations [insets of Fig. 1(b)]. This offers the possibility to investigate the interactions between lattice scatterers as well as with their environment. Another important quantity is the scattering cross section which in this case can be computed using the ratio $\left.\left|\mathbf{E}(\mathbf{r})-\mathbf{E}^{0}(\mathbf{r})\right|^{2} / \mid \mathbf{E}^{0} \mathbf{r}\right)\left.\right|^{2}$, where $\left|\mathbf{E}(\mathbf{r})-\mathbf{E}^{0}(\mathbf{r})\right|^{2}$ is the intensity of the scattered field only. In Fig. 1(c) this ratio is shown for different number of scatterers $N$ in the lattice. It is remarkable that with only 3 scatterers, some specific 


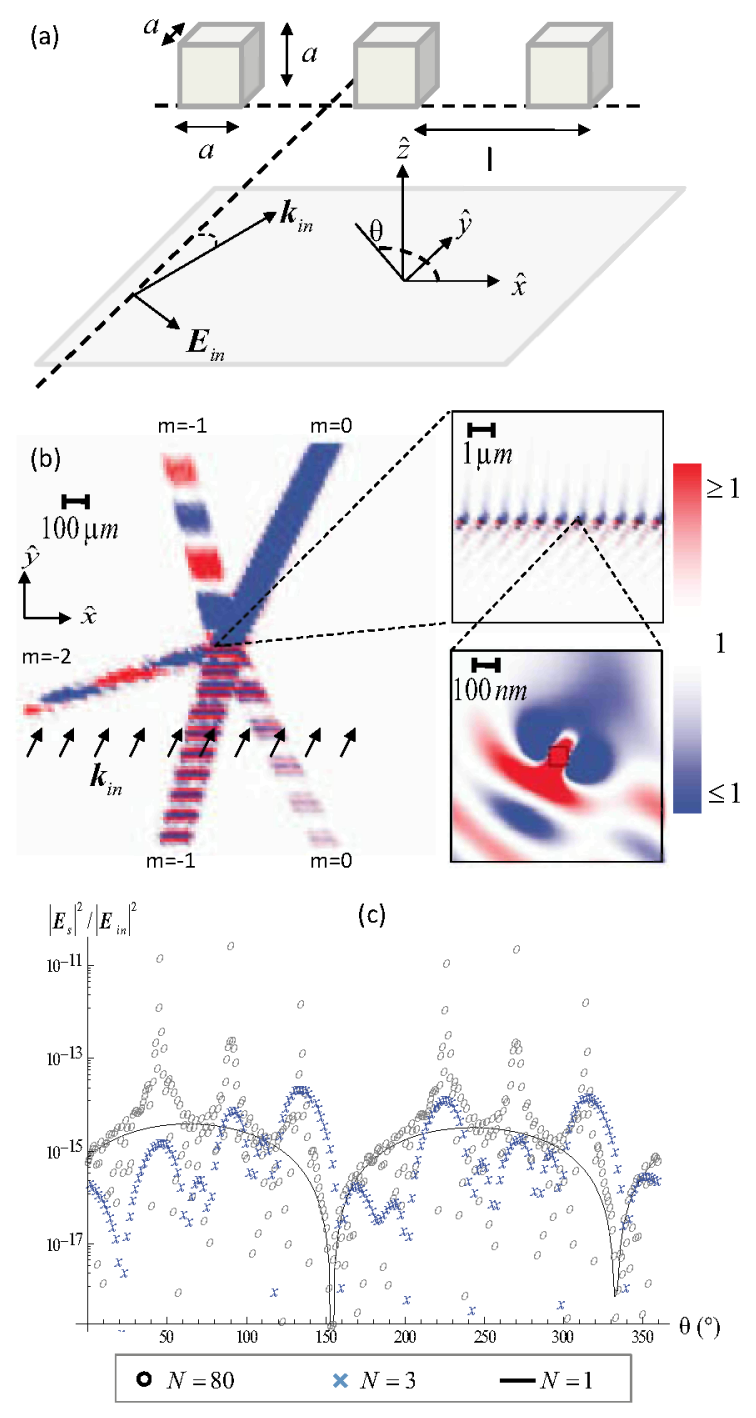

FIGURE 1. Scattering by a periodic array of dielectric cubes ( $\varepsilon=2.25$ ) in air. (a) Scatterers geometry: $a=80 \mathrm{~nm}$ and $l=1000 \mathrm{~nm}$ with $N=80$ scatterers. $\mathbf{k}_{i n}$ and $\mathbf{E}_{i n}$ are respectively the propagation direction and the polarization of the incident plane wave, both with zero $z$ components. The incident wavelength is taken as $2 \pi /\left|\mathbf{k}_{i n}\right|^{2}=700 \mathrm{~nm}$. (b) Total field intensity normalized to the incident field mapped at $z=20 \mathrm{~nm}$ above the cubes edges. The different diffraction orders $m$ are identified. Note that the periodic oscillations are caused by the field sampling. Insets: near field showing a few periods and a single scatterer. (c) Scattering efficiency as a function of the scattering angle $\theta$ for a periodic lattice with $N=1, N=3$ and $N=80$ periods.

scattering directions are already preferred. These directions correspond to plane waves propagating with a wave vector equal to a reciprocal lattice vector $\mathbf{G}$.

More elaborated 3D systems can be simulated with our method, such as a two-dimensional array of scatterers with general lattice translation vectors [Fig. 2]. This

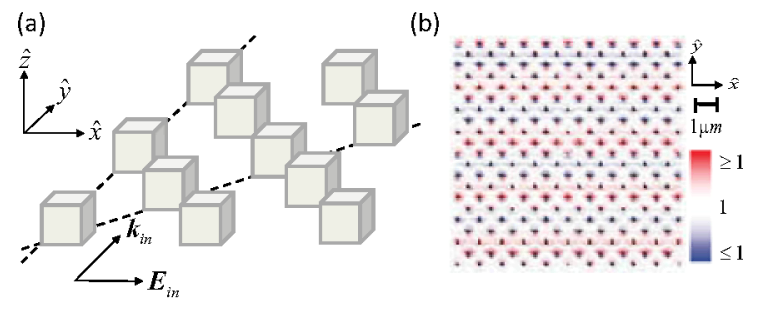

FIGURE 2. Scattering on two-dimensional periodic array of dielectric cubes $(\varepsilon=2.25)$ in air. (a) Sketch of the geometry and the incident plane wave parameters. The incident field is propagating along a primitive lattice vector. (b) Mapping of the total field intensity normalized to the incident field above the lattice.

also holds for anisotropic or metallic scatterers. Both the near and far-field physics can be well investigated, making this method a powerful tool for the design of any nanophotonic system characterized by a periodicity, such as frequency-selective surfaces, photonic band-gap materials, and metamaterials. Furthermore, different approaches to the scatterer discretization can be used, allowing flexibility in the scatterers shape.

\section{ACKNOWLEDGMENTS}

It is a pleasure to acknowledge stimulating discussions with M. Schnieper and A. Stuck. This work was supported by CCMX-SPERU.

\section{REFERENCES}

1. B. T. Draine, and P. J. Flatau, Journal of the Optical Society of America A-Optics Image science and Vision $\mathbf{2 5}$, 2693-2703 (2008).

2. P. C. Chaumet, and A. Sentenac, Journal of Quantitative Spectroscopy \& Radiative Transfer 110, 409-414 (2009).

3. O. Martin, and N. Piller, Physical Review E 58, 3909-3915 (1998).

4. F. Ham, and B. Segall, Physical Review 124, 1786-1796 (1961).

5. C.-T. Tai, Dyadic Green Functions in Electromagnetic Theory, IEEE Series on Electromagnetic Waves, IEEE Press, New York, 1994, 2 edn.

6. I. Stevanovic, P. Crespo-Valero, K. Blagovic, F. Bongard, and J. R. Mosig, IEEE Transactions on Microwave Theory and Techniques 54, 3688-3697 (2006).

7. G. Valerio, P. Baccarelli, P. Burghignoli, and A. Galli, IEEE Transactions on Antennas and Propagation $\mathbf{5 5}$, 1630-1643 (2007).

8. M. Silveirinha, and C. Fernandes, IEEE Transactions on Antennas and Propagation 53, 347-355 (2005).

9. A. Yaghjian, Proceedings of the IEEE 68, 248-263 (1980).

10. P. Flatau, Optics Letters 22, 1205-1207 (1997). 